\title{
GEORG WINTERER
}

\section{Nikotin: \\ Molekulare und physiologische Mechanismen im Zentralen Nervensystem - Ein neues nationales Schwerpunktprogramm der Deutschen Forschungsgemeinschaft}

\section{Einleitung}

Der Senat der Deutschen Forschungsgemeinschaft (DFG) beschloss 2005 die Einrichtung des nationalen Schwerpunktprogramms „Nikotin: Molekulare und physiologische Mechanismen im zentralen Nervensystem“ (SPP1226). Im Jahr 2006 wurden von 50 zur Förderung eingereichten Teilprojekten insgesamt 16 durch ein internationales Expertengremium im Peer-Review-Verfahren für eine Förderung - in Höhe von fünf Millionen € für die erste Förderperiode von drei Jahren - ausgewählt. Als Gesamtlaufzeit sind sechs Jahre vorgesehen. Das Schwerpunktprogramm unter Koordination von Univ.-Prof. Dr. Georg Winterer, Klinik und Poliklinik für Psychiatrie und Psychotherapie der Heinrich-Heine-Universität Düsseldorf, beinhaltet grundlagenwissenschaftliche sowie klinische Projekte (Translational Neuroscience). Tier- und Zellmodelle sind dabei ebenso relevant wie genetische Untersuchungen zur Identifikation erblicher Risikofaktoren, Projekte zur Erstellung und Ergänzung einer großen Multi-Center-Datenbank mit standardisierter Datenerhebung in der Allgemeinbevölkerung und in relevanten Patientengruppen, statistische Projekte zur Methodenentwicklung für die Handhabung komplexer Daten aus der Biomedizin und zu Krankheitsverläufen sowie Studien zur Bildgebung und Physiologie (Endophänotypisierung). Durch eine entsprechende Kombination von Forschungsansätzen soll dabei ein zentrales Ziel des Schwerpunktprogramms, nämlich die genetische und klinische Heterogenität der Nikotinabhängigkeit zu verstehen, erreicht werden.

\section{Ziel und Zweck des Forschungsprogramms}

Ein Drittel aller Erwachsenen weltweit ist Raucher, ${ }^{1}$ und im Jahr 2000 verursachte das Rauchen etwa 2,5 Millionen Todesfälle in den Industrienationen; das sind 19 Prozent der Todesfälle bei Erwachsenen. ${ }^{2}$ Ein besseres Verständnis der zentralnervösen Effekte von Nikotin ist daher aus medizinischer und gesundheitspolitischer Sicht sehr zu wünschen. Das DFG-Schwerpunktprogramm möchte diesem Ziel durch ein Netz aus Grundlagenforschung und klinischer Forschung näher kommen.

\footnotetext{
1 Vgl. World Health Organization (2008).

2 Vgl. Ezzati und Lopez (2003).
} 
Im biopsychologischen Modell der Nikotinabhängigkeit wird davon ausgegangen, dass die hochgradig abhängigkeitserzeugende Wirkung von Nikotin zu einem beträchtlichen Anteil in der neurobiologischen Disposition zur Abhängigkeitsentwicklung verankert ist. Dabei liegt die Vorstellung zugrunde, dass Nikotinabhängigkeit als komplexe, polygene Störung in Wechselwirkung mit Umweltfaktoren zu begreifen ist. So legen Zwillings- und Adoptionsstudien nahe, dass die Erblichkeit für Nikotinabhängigkeit im Bereich von 59 Prozent bei Männern und 46 Prozent bei Frauen liegt. ${ }^{3}$ Von Bedeutung ist auch, dass die Nikotinabhängigkeit kein einheitliches Phänomen darstellt, sondern dass verschiedene Raucher aus unterschiedlichen Gründen Nikotinmissbrauch betreiben (Heterogenität der Erkrankung). Beispielsweise fanden Konkordanzanalysen unter Verwendung verschiedener Maße zur Erfassung der Nikotinabhängigkeit (Konsumfrequenz/Menge, FagerströmTest der Nikotinabhängigkeit, diagnostische Kriterien der Nikotinabhängigkeit und so weiter) heraus, dass die unterschiedlichen Maße teilweise voneinander unabhängige Aspekte der Nikotinabhängigkeit erfassen. ${ }^{4}$ Alkoholkonsum, bestimmte Lifestyle- und Umweltfaktoren, Persönlichkeitsmerkmale wie zum Beispiel Abhängigkeit von belohnenden Verstärkern und schließlich psychiatrische Störungen (Dual Diagnosis: zum Beispiel Depression, Aufmerksamkeitsstörung, Schizophrenie) sind teilweise unabhängig voneinander mit Nikotinabhängigkeit korreliert. ${ }^{5}$ Einige der letztgenannten Faktoren können sich außerdem während der Abstinenz verändern (zum Beispiel Gewichtszunahme, Appetit, depressive Stimmung, erhöhte Stressanfälligkeit, kognitive Defizite, Schlafstörungen), was wiederum einen Rückfall provozieren kann. In vorangegangenen genetischen Studien der Nikotinabhängigkeit wurde diese Heterogenität der Nikotinabhängigkeit üblicherweise nicht berücksichtigt. Dies mag ein Grund dafür sein, dass bislang kein Gen konsistent mit Nikotinabhängigkeit assoziiert werden konnte (Mangel an statistischer Power).

In dem gerade begonnen Schwerpunktprogramm der DFG wird die Heterogenität der Nikotinabhängigkeit sowohl in den grundlagenwissenschaftlichen als auch in den klinischen Forschungsprojekten berücksichtigt werden. Beispielsweise geschieht dies in den klinischen Studien unter Verwendung der so genannten Endophänotypisierungsstrategie. ${ }^{6}$ Bei diesem Forschungsansatz werden nicht nur Gene von (abhängigen) Rauchern und Nichtrauchern verglichen, sondern auch nikotinabhängigkeitsbezogene neurobiologische Phänotypen wie zum Beispiel Craving, kognitive Veränderungen, stressbezogene hormonelle Veränderungen und so weiter untersucht (sekundäre Endpunkte). Unter anderem wird durch die Anwendung dieser Forschungsstrategie erwartet, dass daraus ein besseres Verständnis der neurobiologischen Mechanismen einschließlich der Beteiligung einzelner Gene (sowie deren Interaktion mit Umweltfaktoren) der Nikotinabhängigkeit resultiert. In diesem Zusammenhang ist es von besonderer Bedeutung, dass komplementär zu und in enger Abstimmung mit den klinischen Studien experimentelle, grundlagenwissenschaftliche Studien durchgeführt werden (Translational Neuroscience). Zwar liegt während der ersten Förderperiode das Schwergewicht auf klinischen Forschungsprojekten, es ist aber beabsichtigt, dass während der zweiten Förderperiode grundlagenwissenschaftliche Untersuchungen erheblich stärker berücksichtigt werden. Dabei sollen auf der Basis der in den

\footnotetext{
3 Vgl. Li et al. (2003) sowie Vink et al. (2005).

4 Vgl. Moolchan et al. (2002), Hughes et al. (2004) sowie Furberg et al. (2005).

5 Vgl. zum Beispiel Hertling et al. (2005), Whalen et al. (2002) sowie Rukstalis et al. (2005).

6 Vgl. Winterer et al. (2005).
} 
klinischen Studien gewonnen Erkenntnisse zu den zentralen neurobiologischen Systemmechanismen der Nikotinabhängigkeit die beteiligten Gene identifiziert und näher charakterisiert werden beziehungsweise diese zum Beispiel in transgenen Zell- beziehungsweise Tiermodellen experimentell auf ihre Funktionseigenschaften überprüft werden.

Es werden folgende Themenbereiche bearbeitet werden:

- molekulargenetische Mechanismen der Nikotinabhängigkeit, auch mit Berücksichtigung von Gen-Umwelt-Interaktion;

- klinische Charakterisierung (Phänotypisierung) großer Multi-Center-Stichproben aus der Allgemeinbevölkerung und von neuropsychiatrischen Patienten mit Berücksichtigung von Veränderungen während des Entzugs und Bestimmung von Risikofaktoren für den Rückfall;

- funktionelle Analysen der physiologischen Effekte von Nikotin und Nikotinentzug im Gehirn durch Bildgebung und endokrinologische Studien.

Im Einzelnen wurden folgende Projekte in das Schwerpunktprogramm aufgenommen:

Koordinationsprojekt:

„Nikotin: Molekulare und physiologische Effekte im zentralen Nervensystem“

Univ.-Prof. Dr. Georg Winterer, Klinik und Poliklinik für Psychiatrie und Psychotherapie, Heinrich-Heine-Universität Düsseldorf

Grundlagenwissenschaftliche Forschungsprojekte:

„Identifizierung funktioneller nAChR-Varianten und ihre Rolle bei Nikotinabhängigkeit, Schizophrenie und Epilepsie“"

Univ.-Prof. Dr. Ortrud Steinlein, Institut für Humangenetik, Klinikum der LudwigMaximillians-Universität München

Prof. Dr. Daniel Bertrand, Department des Neurosciences fondamentales, Université de Genève, Schweiz

„Nikotin, synaptische Plastizität und Abhängigkeit: Molekulare Mechanismen der Nikotin-induzierten funktionellen Aufregulation des nACh-Rezeptors alpha4beta2“

PD Dr. Karl-Heinz Braunewell, Neurowissenschaftliches Forschungszentrum, Charité - Universitätsmedizin Berlin

„Die Rolle des Cannabinoid-Rezeptors CB1 in der Nikotinabhängigkeit““

Univ.-Prof. Dr. Beat Lutz, Institut für Physiologische Chemie und Pathobiochemie, Johannes-Gutenberg-Universität Mainz

Klinische Forschungsprojekte:

„Genetik der Nikotinabhängigkeit: Klinische und neurobiologische Phänotypen in einer multizentrischen Fall-Kontrollstudie“"

Univ.-Prof. Dr. Georg Winterer, Klinik und Poliklinik für Psychiatrie und Psychotherapie, Heinrich-Heine-Universität Düsseldorf

Univ.-Prof. Dr. Thomas Wienker, Institut für Medizinische Biometrie, Informatik und Epidemiologie, Rheinische Friedrich-Wilhelms-Universität Bonn 
Univ.-Prof. Dr. Falk Kiefer, Zentralinstitut für Seelische Gesundheit, Mannheim PD Dr. Michael Wagner, Klinik und Poliklinik für Psychiatrie und Psychotherapie, Rheinische Friedrich-Wilhelms-Universität Bonn

Univ.-Prof.Dr. Gerd Gründer, Klinik für Psychiatrie und Psychotherapie, Rheinisch-Westfälische Technische Hochschule Aachen

PD Dr. Jürgen Gallinat, Klinik und Poliklinik für Psychiatrie und Psychotherapie, Charité Berlin

Assoziierte Zentren:

Dr. habil. Thomas Sander/Univ.-Prof. Dr. Peter Nürnberg, Cologne Center for Genomics (CCG), Universität zu Köln

Dr. Norbert Thürauf, Psychiatrische und Psychotherapeutische Klinik, FriedrichAlexander-Universität Nürnberg-Erlangen

Dr. Stefan Cohrs/PD Dr. Rodenbeck, Klinik und Poliklinik für Psychsomatik und Psychotherapie, Georg-August-Universität Göttingen

„Analyse neuer Kandidatengene der Nikotinabhängigkeit anhand einer genomweiten Assoziationsstudie“"

PD Dr. Norbert Dahmen, Klinik und Poliklinik für Psychiatrie und Psychotherapie, Johannes-Gutenberg-Universität Mainz

Univ.-Prof. Dr. Georg Winterer, Klinik und Poliklinik für Psychiatrie und Psychotherapie, Heinrich-Heine-Universität Düsseldorf

Dr. Dan Rujescu, Klinik für Psychiatrie und Psychotherapie, Klinikum der Ludwig-Maximillians-Universität München

Univ.-Prof. Dr. Andreas Gal, Institut für Humangenetik, Zentrum für Frauen-, Kinder- und Jugendmedizin, Universitätsklinikum Hamburg-Eppendorf

Univ.-Prof. Dr. Heinz-Erich Wichmann, Forschungszentrum für Umwelt und Gesundheit (GSF), Institut für Entwicklungsgenetik, Oberschleißheim

„Genetische Faktoren und Gen-Umwelt-Wechselwirkungen permanenter Abstinenz bei stark abhängigen Rauchern: Retrospektive Kohortenanalysen von 1.553 stark abhängigen Rauchern“"

Univ.-Prof. Dr. Hermann Brenner, Deutsches Krebsforschungsinstitut Heidelberg, Abteilung für Klinische Epidemiologie und Alternsforschung

„Aufmerksamkeitsnetzwerk, Nikotinabhängigkeit und nACh-alpha4beta2-Rezeptor-Genotyp bei Gesunden und schizophrenen Patienten“

Univ.-Prof. Dr. Georg Winterer, Institut für Medizin, Forschungszentrum Jülich/ Klinik und Poliklinik für Psychiatrie und Psychotherapie, Heinrich-Heine-Universität Düsseldorf

Univ.-Prof. Dr. Gereon Fink, Institut für Medizin, Forschungszentrum Jülich/Neurologische Klinik der Universität zu Köln

„Dopamin-Metabolismus bei Nikotinabhängigkeit: [18F]FDOPA-PET- und fMRT-Studien bei rauchenden und entgifteten Personen“ 
Univ.-Prof. Dr. Gerhard Gründer, Klinik für Psychiatrie und Psychotherapie, Rheinisch-Westfälische Technische Hochschule Aachen

Univ.-Prof. Dr. Udalrich Büll, Klinik für Nuklearmedizin, Rheinisch-Westfälische Technische Hochschule Aachen

Univ.-Prof. Dr. Thilo Kircher, Klinik für Psychiatrie und Psychotherapie, Rheinisch-Westfälische Technische Hochschule Aachen

Dr. Dirk Leube, Klinik für Psychosomatik und Psychotherapeutische Medizin, Rheinisch-Westfälische Technische Hochschule Aachen

Dr. Ingo Vernaleken, Klinik für Psychiatrie und Psychotherapie, Rheinisch-Westfälische Technische Hochschule Aachen

„Effekte von Geschlecht und Nikotinabhängigkeit auf die Funktion der Amygdala (Mandelkern) beim Menschen während sozialer Interaktion“"

Dr. Rene Hurlemann, Klinik und Poliklinik für Psychiatrie und Psychotherapie, Rheinische Friedrich-Wilhelms-Universität Bonn

„Risikoprädiktion der Nikotinabhängigkeit auf der Basis der Untersuchung individueller Grenzen kortikaler Neuroplastizität beim Menschen“"

Dr. Michael Nitsche, Abteilung für klinische Neurophysiologie, Georg-AugustUniversität Göttingen

Dr. Nicolas Lang, Abteilung für klinische Neurophysiologie, Georg-August-Universität Göttingen

Univ.-Prof. Dr. Walter Paulus, Abteilung für klinische Neurophysiologie, GeorgAugust-Universität Göttingen

„Beendigung des Nikotinmissbrauchs und Entzugserscheinungen: Veränderungen neuroendokrinologischer und polysomnographischer Parameter als Rückfallprädiktoren“

Univ.-Prof. Dr. Dieter Riemann, Abteilung für Psychiatrie und Psychotherapie, Universitätsklinikum Freiburg

PD Dr. Andrea Rodenbeck, Klinik und Poliklinik für Psychosomatik und Psychotherapie, Georg-August-Universität Göttingen

Dr. Stefan Cohrs, Klinik und Poliklinik für Psychosomatik und Psychotherapie, Georg-August-Universität Göttingen

Univ.-Prof. Dr. Stefan Andreas, Fachklinik für Lungenerkrankungen, Immenhausen

„Nikotineffekte auf emotionale und motivationale Verarbeitung“

Dr. Michael Smolka, Zentralinstitut für Seelische Gesundheit Mannheim

Dr. Alexander Diehl, Zentralinstitut für Seelische Gesundheit Mannheim

„Ein stereospezifisches chemosensorisches Modell zur Analyse der Nikotinwahrnehmung und sensorisch induzierten Cravings

Dr. Norbert Thürauf, Psychiatrische und Psychotherapeutische Klinik, FriedrichAlexander-Universität Erlangen-Nürnberg 
Univ.-Prof.Dr. Stefan Bleich, Psychiatrische und Psychotherapeutische Klinik, Friedrich-Alexander-Universität Erlangen-Nürnberg

Dr. Thilo Hammen, Radiologisches Institut, Friedrich-Alexander-Universität Erlangen-Nürnberg

Univ.-Prof. Dr. Johannes Kornhuber, Psychiatrische und Psychotherapeutische Klinik, Friedrich-Alexander-Universität Erlangen-Nürnberg

„Nikotineffekte auf Endophänotypen der Schizophrenie“

PD Dr. Michael Wagner, Klinik und Poliklinik für Psychiatrie und Psychotherapie, Friedrich-Wilhelms-Universität Bonn

Dr. Christian Schütz, Klinik und Poliklinik für Psychiatrie und Psychotherapie, Friedrich-Wilhelms-Universität Bonn

„Nikotineffekte auf soziale Kognition und sozialen Stress bei Schizophrenie“

Univ.-Prof. Dr. Wolfgang Wölwer, Klinik und Poliklinik für Psychiatrie und Psychotherapie, Heinrich-Heine-Universität Düsseldorf

Univ.-Prof. Dr. Georg Winterer, Institut für Medizin, Forschungszentrum Jülich/ Klinik und Poliklinik für Psychiatrie und Psychotherapie, Heinrich-Heine-Universität Düsseldorf

Weitere Informationen zum DFG-Schwerpunktprogramm finden Sie auf der Website http://www.nicotine-research.com beziehungsweise erteilt der Koordinator des Schwerpunktprogramms, Univ.-Prof. Dr. Georg Winterer, Heinrich-Heine-Universität Düsseldorf, Klinik und Poliklinik für Psychiatrie und Psychotherapie, Bergische Landstraße 2, 40629 Düsseldorf, Tel. 0211/9223495, E-Mail: georg.winterer@uni-duesseldorf.de

\section{Literatur}

EzZATI, M. und A. D. Lopez (2003). „Estimates of global mortality attributable to smoking in 2000“, Lancet 362, 847-852.

Furberg, H., P. F. Sullivan, H. Maes, C. A. Prescott, C. Lerman, C. Bulik und K. S. KENDLER (2005). „The types of regular cigarette smokers: A latent class analysis“, Nicotine Tobacco Research 7, 351-360.

Hertling, I., K. Ramskogler, A. Dvorak, A. Klingler, G. Saletu-Zyhlarz, R. Schoberberger, H. Walter, M. Kunze und O. M. Lesch (2005). „Craving and other characteristics of the comorbidity of alcohol and nicotine dependence“, European Psychiatry $20,442-450$.

Hughes, J. R., A. H. Oliveto, R. Riggs, M. Kenny, A. Liguori, J. L. Pillitteri, M. A. MACLAUGHLIN (2004). „Concordance of different measures of nicotine dependence: two pilot studies“, Addictive Behavior 29, 1527-1539.

LI, M. D., R. Cheng, J. Z. MA und G. E. SWAn (2003). „A meta-analysis of estimated genetic and environmental effects on smoking behavior in male and female adult twins“, Addiction 98, 23-31.

Moolchan, E. T., A. Radzius, D. H. Epstein, G. Uhl, D. A. Gorelick, J. L. Cadet, J. E. HenningField (2002). ,The Fagerstrom Test for Nicotine Dependence and the Diagnostic Interview Schedule: do they diagnose the same smokers?", Addictive Behavior 27, 101-113. 
Rukstalis, M., C. Jepson, F. PATterson und C. Lerman (2005). „Increases in hyperactiveimpulsive symptoms predict relapse among smokers in nicotine replacement therapy“, Journal of Substance Abuse and Treatment 28, 297-304.

VINK, J. M., G. WILLEMSEN und D. I. BOOMSMA (2005). „Heritability of smoking initiation and nicotine dependence“, Behavior Genetics 35, 397-406.

Whalen, C. K., L. D. Jamner, B. Henker, R. J. Delfino und J. M. Lozano (2002). „The ADHD spectrum and everyday life: experience sampling of adolescent moods, activities, smoking, and drinking“, Child Development 73, 209-227.

Winterer, G., A. R. HARIRI, D. Goldman und D. R. Weinberger (2005). „Neuroimaging and human genetics“, International Reviews of Neurobiology 67, 325-383.

World Health ORganization (2008). WHO Report on the Global Tobacco Epidemic, 2008 - The MPOWER package. http://www.who.int/tobacco/mpower/mpower_report_full_2008.pdf (06.11.2008). 
